\title{
Adaptive Extensions of Object-Oriented Systems
}

\author{
Olaf Seng, Thomas Genssler, Benedikt Schulz \\ Forschungszentrum Informatik \\ Haid-und-Neu-Straße 10-14, 76131 Karlsruhe, Germany \\ $\{$ seng, genssler, bschulz $\} @$ fzi.de
}

\begin{abstract}
In order to satisfy the changing needs of customers and clients, software systems have to be adapted during their whole lifetime. One frequently occurring task is adding a new function to the interface of a (sub-) system. To implement the new functionality, not only the interface has to be extended but several other classes of the (sub-) system. Performing this task manually is tedious and error-prone. In this paper a method is described that simplifies implementing extensions of the above kind. The basic idea is using adaptive programming (AP), so that new functionality can be described with the help of paths in the class structure and source code transformations.
\end{abstract}

Keywords: Software re-engineering, Adaptive Programming, Scripting languages

\section{Introduction}

\subsection{Context}

Software engineering is an evolutionary process. Extensions to existing systems are necessary, since requirements change, new ones emerge, or old ones have just not been implemented correctly. Such extensions do not only occur during forward engineering, like in Extreme Programming (XP) [2], but are also part of software maintenance and re-engineering [15].

In this paper, only those extensions to object-oriented systems are considered, where a new function has to be added to the interface of a subsystem $^{1}$. The interface could be for example a facade, according to

\footnotetext{
${ }^{1}$ In this paper, any set of classes is called a subsystem
}

The original version of this chapter was revised: The copyright line was incorrect. This has been corrected. The Erratum to this chapter is available at DOI: 10.1007/978-0-387-35672-3_13 


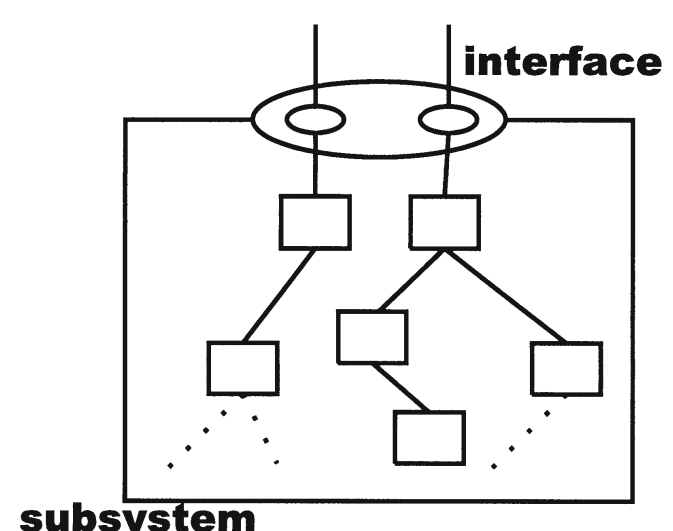

Figure 1. View of existing subsystem

the design pattern from [5]. Very often, it is not sufficient to just modify the interface. Further modifications of a couple of classes not being part of the interface of the system have to be performed. Figure 1 shows an imaginary example of such a situation. Two functions are available at the interface of the subsystem. The classes - drawn as boxes - that are needed to implement the respective function are interconnected by lines. These lines represent interactions between the classes.

Taking a closer look to the classes involved in providing the functionality of the new function, it is observable that there are two categories of classes. Classes of the first category are just forwarding messages to other involved classes, which is done by simple method calls. Classes of the second category additionally contain source code that performs the required computations. This claim has been checked by [17] during several case studies. It has been discovered that $50 \%$ of all methods contained fewer than four statements. This property of object oriented systems is called the small method problem. In the next section we will describe, why it is hard to implement extensions with so many small methods.

\subsection{Problem}

Figure 2 shows the typical process of carrying out the extensions. At first, the programmer has to build a model view of the system to be extended. Otherwise he is not able to plan his work. In rare cases such a model might be already existing. It should provide a more abstract view of the source code, that does not contain all implementation details. 


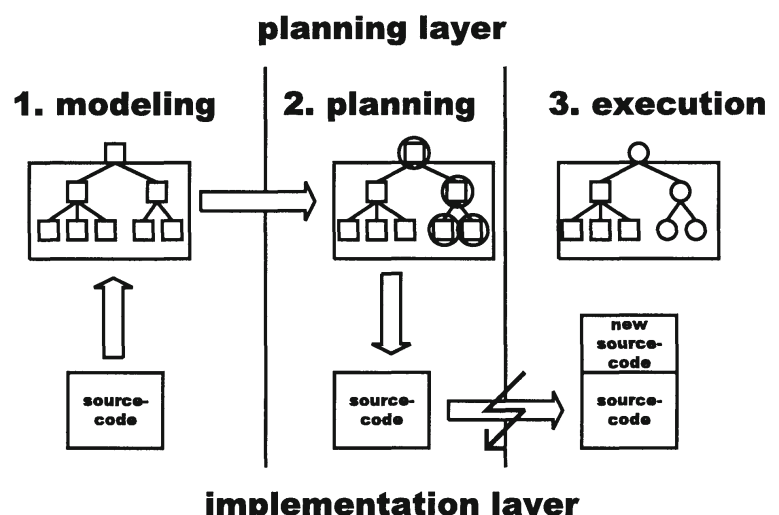

Figure 2. State of the art in performing the described extensions

During the planning phase the engineer has to identify the classes which are going to be involved in the extension. He has to identify those classes, that only need to perform some message delegation via function calls, and those in which source code performing actual computations has to be inserted.

The execution of the planned steps takes place on the low implementation level. Every class has to be modified manually. And this leads to our problem: since many classes are involved and the detailed whitebox view must be maintained during modification, this task gets very difficult and error-prone.

\subsection{Goal and Solution}

For systems, that are constructed from scratch, Adaptive programming (AP) [10] offers a powerful way for solving the small method problem by automatically generating those methods that just forward messages to other classes. The aim of our work is to provide a procedure, which is capable of performing the described extensions in a suitable way during forward engineering and re-engineering. The starting point is checking whether existing approaches, especially AP can be used as a basis for the method. In order to make the new method useful in practice, a supporting prototypical tool should be developed.

Figure 3 shows the basic idea for an improvement of the standard procedure. The developer should be able to specify the extensions on the same abstract level, on which he has already planned them. Not all of the implementation details should have to be mentioned during 


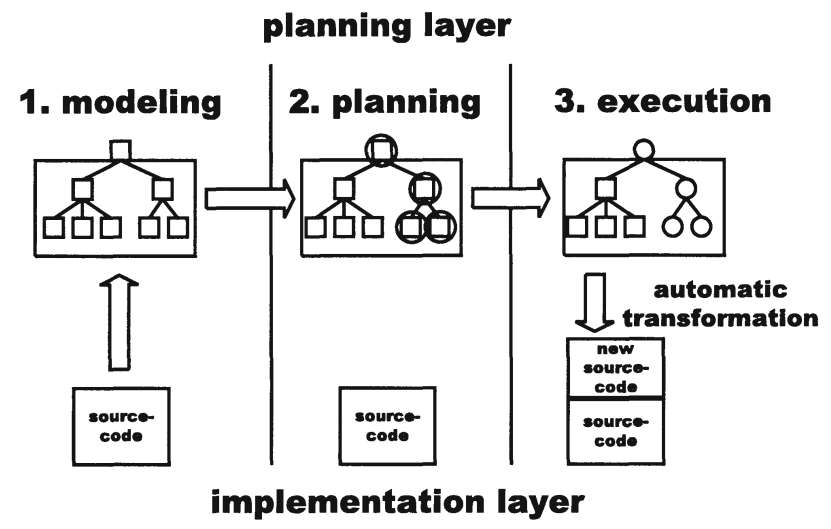

Figure 3. Improved way of performing the extensions

specification, a grey-box view of the system should be sufficient. This specification should be automatically transformed to source code.

Such a method should satisfy the following criteria:

- Scalability: Systems of any size should be processable. The size is determined by lines of code. It should be no problem to deal with systems with millions of lines of code.

- Universality: The method should be able to process existing, objectoriented source code.

- Degree of abstraction: White-box-knowledge should not be necessary for specification and execution of the extension.

- Expressiveness: Compared to standard procedures, the method should be more expressive, which can be measured by the amount of written lines of code.

- Source code quality: The code of the system after applying the method should be maintainable, that is readable and expandable.

The rest of this paper is organized as follows. In section 2, existing methods to perform extensions are presented and evaluated considering the above criteria. The new approach is presented in section 3 , followed by a short description of the implementation in section 4 . Section 5 evaluates the new method by first presenting one case study and then examining if the method fulfill the desired criteria. The paper finishes in section 6 with a short summary and some suggestions for further work. 


\section{State of the art}

This section presents existing methods that are used today to perform the described extensions. After a short description the methods are evaluated considering the criteria from section 1 .

\subsection{Manual approach}

The manual approach is the normal procedure for modifying existing code. Developers use integrated development environments like for example Together $^{2}$, JBuilder ${ }^{3}$ or just a simple text editor and a compiler. The planned extensions have to be performed directly on the implementation level, by navigating to each affected class and adding new code. During this step the developer must be aware of all implementation details, so he has to maintain the white box view of the system. Obviously such a procedure does not fulfill the criterion degree of abstraction.

This approach might work for small systems, but trying to extend large systems will be even harder, since it is getting more and more difficult to keep track of the classes that have already been modified and those that must still be modified. So the criterion scalability is not fulfilled.

\subsection{Refactorings}

Refactorings are designed for improving the structure of existing systems [12] [4]. They consist of formal specified operations, like createEmptyClass or deleteMethod. It would be possible to misuse refactorings by not only modifying the structure but adding new functionality to a given system. Some existing refactorings could be used instantly and some others might have to be invented, for example one that adds some portion of code to a given method.

Applying refactorings would be a more formal way for the developer of implementing the considered extensions, but since each step would have to be described with a single refactoring such a procedure is not more expressive than doing it manually. So the criterion expressiveness is not fulfilled.

Using refactorings does not fulfill the criterion degree of abstraction, since the user must deal with all implementation details while specifying the extensions with the refactorings.

\footnotetext{
${ }^{2}$ http://www.togethersoft.com

${ }^{3}$ http://www.inprise.com
} 


\subsection{Adaptive Programming (AP)}

$\mathrm{AP}$ is a new kind of paradigm to software engineering, closely related to Aspect Oriented Programming (AOP) [8]. Being an extension to conventional object-oriented programming, its main difference is the separate specification of structure and functionality of a program. The structure of a program is defined by a class graph which is a kind of UML class diagram. The functionality is specified by propagation patterns. A propagation pattern contains a navigation directive and several code injections. Navigation directives are written as paths in the class graph, consisting of a starting class and some end classes.

A code injection specifies a couple of code statements and the classes in which the statements should be executed, as soon as those classes are reached during navigation.

Considering the properties of $\mathrm{AP}$, it is clear that using $\mathrm{AP}$ concepts will fulfill some of the criteria the new method should achieve. The extension to our subsystem could be written in form of a propagation pattern, where the communication in terms of method calls can be defined as a navigation directive.

- Scalability: To specify the extensions, it is only necessary to have a local view on the part of the system that is going to be extended during the specification. This is a must for really large systems since one cannot have the whole system in mind.

- Degree of abstraction: AP programs are written on a more abstract level than conventional ones. Not all implementation details have to be specified.

- Expressiveness: Writing an extension as a propagation pattern needs fewer instructions, since navigation from one class to another is automatically calculated and inserted into source code.

Currently there are several versions of AP, which are supported by tools and might be useful. The first one is DemeterJ [9]. Translatable Java programs are generated from a specification of a class diagram and several propagation patterns. The problem is, that this method was not designed to handle existing code, but to construct new systems. So this approach cannot fulfill the desired criterion universality.

A newer approach called DJ [13], is more likely to be used together with existing systems. The class graph does not need to be specified separately from the program, but can be extracted from the code using reflection. The AP concepts are provided as a library, so that programs can be augmented with propagation patterns easily by using Java constructs. 


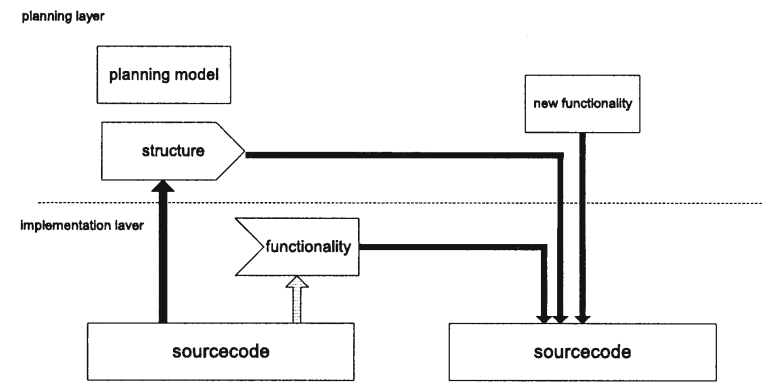

Figure 4. Overview of the new approach

Using reflection leads to a loss of performance, since class graph generation and calculating the navigation paths take place at runtime after all classes of the systems have been loaded. Another drawback of this approach is, that the code resulting from an application must be shipped with the library and contains statements not understandable by a programmer without AP knowledge. So readability is getting worse and programs cannot be evolved and translated without the library, so maintainability has decreased. As a consequence this approach cannot satisfy the criterion source code quality.

Two other approaches exist that make use of AP. The first one is a students' project at the Northeastern University called TraversalJ [11], a recently started work that enhances AspectJ [7] with adaptive capabilities. The projects seems to be not completed yet and no detailed documents describing the features of this tool are available, so it cannot be decided whether this approach satisfies the desired criteria.

The second approach using AP has been suggested by Michael Werner in his $\mathrm{Ph} . \mathrm{D}$. thesis [16]. He calls propagation patterns itineraries and mentions that they can be attached to existing systems. Since he does not show any evaluation and practical experiences of his ideas, it cannot be judged whether his approach can fulfill our criteria.

\section{Our approach: Extending systems adaptively}

The main idea of the new approach is using AP as a basis which is modified and extended, so that all the desired criteria are fulfilled and AP can be used as part of evolutionary software development.

Figure 4 presents our process of adding new functionality to an interface: At first a model of the existing source code is extracted, that is building the class graph, to get a more abstract view of the system. The 


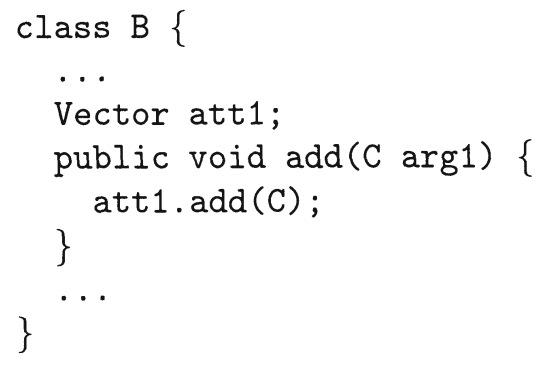

Figure 5. Class, using a Java collection class.

user plans the extensions with respect to this model. He identifies the classes to be altered, and he determines the pieces of code to be executed in those classes.

Having completed the planning step the new approach allows the user to specify the planned extension apart the existing code in one or more metaprograms ${ }^{4}$ using AP. This allows writing the extension on an abstract level without all implementation details.

The metaprograms are going to be evaluated before compile time. New source code is automatically constructed from the three existing parts, the structure, the existing functionality and the metaprograms.

In order to deal with every given object-oriented system and ensuring the claimed quality of source code, the AP approach has to be modified and improved. The first modification concerns the model, describing the entities and relations, that can be inserted into a given class graph. The model from [10] serves as a basis, but has to be changed in order to reflect super classes violating the abstract-super-class-rule [6]. In our model super classes do not have to be abstract and are allowed to have attributes, since existing systems will contain such super classes.

The creation of the class graph has to be modified too. Since propagation patterns are evaluated before run-time, the calculation of the propagation paths relies on the information statically available from the source code. This leads to paths that will never exist at run time, since the types of some class attributes are too general.

An example are the so called collection classes in Java, which are able to store objects of type java. lang. Object in order to process each existing Java class.

\footnotetext{
${ }^{4} \mathrm{~A}$ metaprogram is a program modifying itself or other programs [3]
} 
Consider the code in Figure 5. It is obvious that class B is only storing objects of type $\mathrm{C}$ in its Vector. A path containing such a class $\mathrm{B}$ will possibly lead to all classes of the system, since java.lang.Object is the direct or indirect base class of all other classes. This might lead to modifications of classes, that are not planned.

As a consequence a pre-processing type inference step has to be added in front of the class graph generation, which tries to determine the types of the objects at run-time. Examples for type inference algorithms are explained in [1] or [14].

The language features of the metaprograms are a suitable selection of existing ones. They allow all main AP constructs to be expressed, like propagation patterns, navigation directives, transportation of variables, ... The selection tries to simplify the use of AP constructs without reducing expressiveness.

To ensure the desired code quality, the generated code should look like code written by a human. This is achieved by creating a new method in each class and inserting the code injections and calls to other methods directly in the newly constructed methods.

\section{Implementation}

The new approach has been realized with Inject/ $\mathrm{J}^{5}$ for Java as a prototypical implementation, to gain some practical experiences. Inject/J is a tool for adapting existing software systems, that have to be modified due to some changes of requirements. It offers a scripting language letting the user specify adaptations and is able to transform source code according to the scripts.

Inject/J has been used for two reasons. At first, it provides much of the required functionality like model extraction, adding new classes and new methods, adding new portions of source code to existing methods etc. which simplifies the implementation of the new approach. At second the new approach fits well into Inject/J, since adding new functionality to an existing interface can also be seen as a kind of adaptation.

To give a short impression how an Inject/J script with AP features looks like, a small example is presented in Figure 6.

The script starts with identifying the script as script SimpleExample, which is useful in case of having written more than one script and wishing to specify the order of execution.

The in class 'A' .. to ' $C$ ' part specifies the navigation directive of the propagation pattern. The navigation starts in class A and

${ }^{5}$ http://injectj.fzi.de 


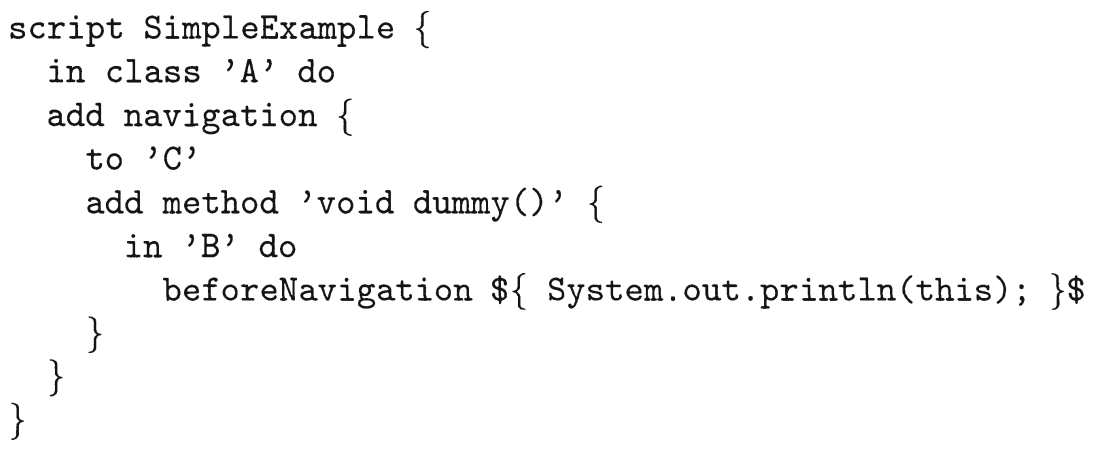

Figure 6. Inject/J example with propagation pattern

leads to class $\mathrm{C}$ traversing all classes being part of the paths in the class graph from A to C. By add method ... the name of the method being added to the interface of class $\mathrm{A}$ is defined. The following part in ' $\mathrm{B}$ ' do ... specifies some functionality that should be executed as part of the propagation pattern. When the navigation reaches class B the Java code contained between $\$\{$ and $\} \$$ is executed before navigating any further.

The execution of this script will be done as follows. After starting the Inject/J tool, the user has to enter the location of his source code, so that Inject/J can build its interior model, the class graph. Next, the script to be executed has to be selected by the user. Afterwards the script is interpreted and the source code is transformed according to the instructions contained in the script.

In the current implementation, the type inference is carried out during a very simple precalculating step. For each attribute a, that is a collection class, all calls to methods which add elements to a are looked up in the source code. Those calls have the following form: a.<addMethodName>(<expression>). Using a type inference algorithm the set of possible types of <expression> can be calculated. The obtained information is stored in source code as comments, enabling the user to modify and refine it afterwards. Of course this step cannot capture all types that are contained in the collection class attributes at run time. But it turned out that in all three examined case studies the content of about 80 per cent of those attributes could be identified. The other 20 per cent consisted of collection class attributes for which no add method could be found in the source code. 


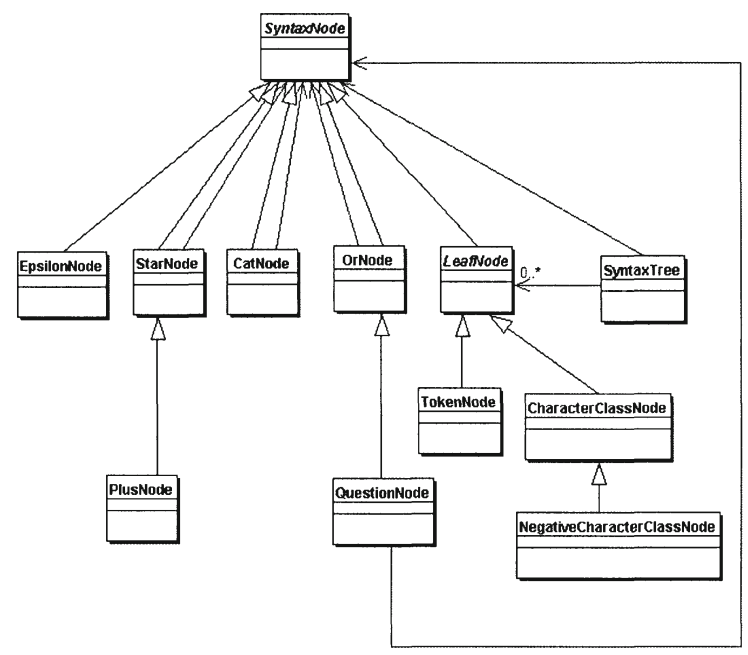

Figure 7. Existing part of ORO

\section{Evaluation}

The evaluation starts describing some practical experiences applying the new method to an existing system and ends with a general discussion whether the claimed criteria can be fulfilled or not.

\subsection{Case study}

The selected case study represents a system, whose source code is freely available and which is written in Java, since the tool only supports this language right now. Any performed extension is artificial, but typical in the way it affects classes and methods of the system.

5.1.1 ORO. In a first attempt, ORO - part of the Apache Jakarta project ${ }^{6}$ - has been extended. ORO contains a set of text processing classes, which enable support for regular expressions in JAVA being compatible with Perl5 or AWK. In this example the part of the system responsible for AWK expression has to be extended. The structure of this part is shown as a class graph in Figure 7. It has been generated from the source code. Classes are drawn as boxes, attribute relations are painted with small arrow heads, inheritance relations are

${ }^{6} \mathrm{http}: / /$ jakarta.apache.org 


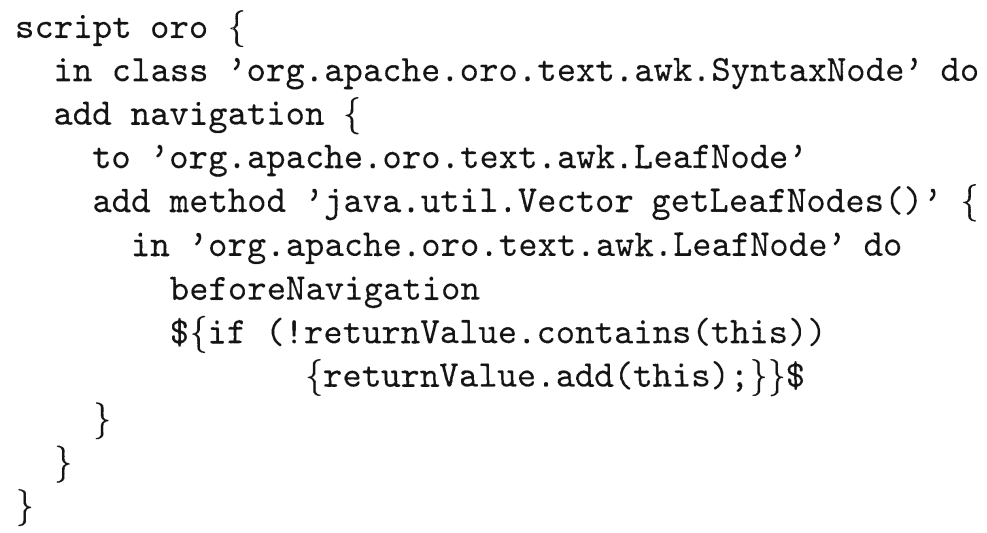

Figure 8. Script for extension of ORO

expressed via large heads. The diagram shows, that regular expressions are represented as abstract syntax trees.

Now a new function should be added to each node type of the syntax tree, that returns the leaf nodes available from the current node. One possible implementation of such a function uses a recursive approach. From the node on which the operation has been called a traversal to all reachable objects of type LeafNode starts. If such an object has been reached, it inserts itself into a transport variable and returns to its parent node. The respective script is presented in Figure 8.

One new feature of the AP scripting language can be observed from the above piece of code. Some kind of variables that are global with respect to the path can be used during traversal of that path. This feature represents the transportation patterns of AP. In this example, a variable of the return type java.util.Vector with the name returnValue can be accessed from every class involved.

Figure 9 shows the result of an application of the script from above. One can see the new methods in the node types. The creation of two methods with different signatures is necessary in order two have a possibility to transport the global variables along the navigation paths.

This example shows the expressiveness of the new approach. The method takes care of the navigation to the leaf nodes and the corresponding source code is automatically generated. By not having to take care for all implementation details - only two of the involved classes have to be specified - it is obvious, that the method offers a higher degree of abstraction than writing all code manually. 


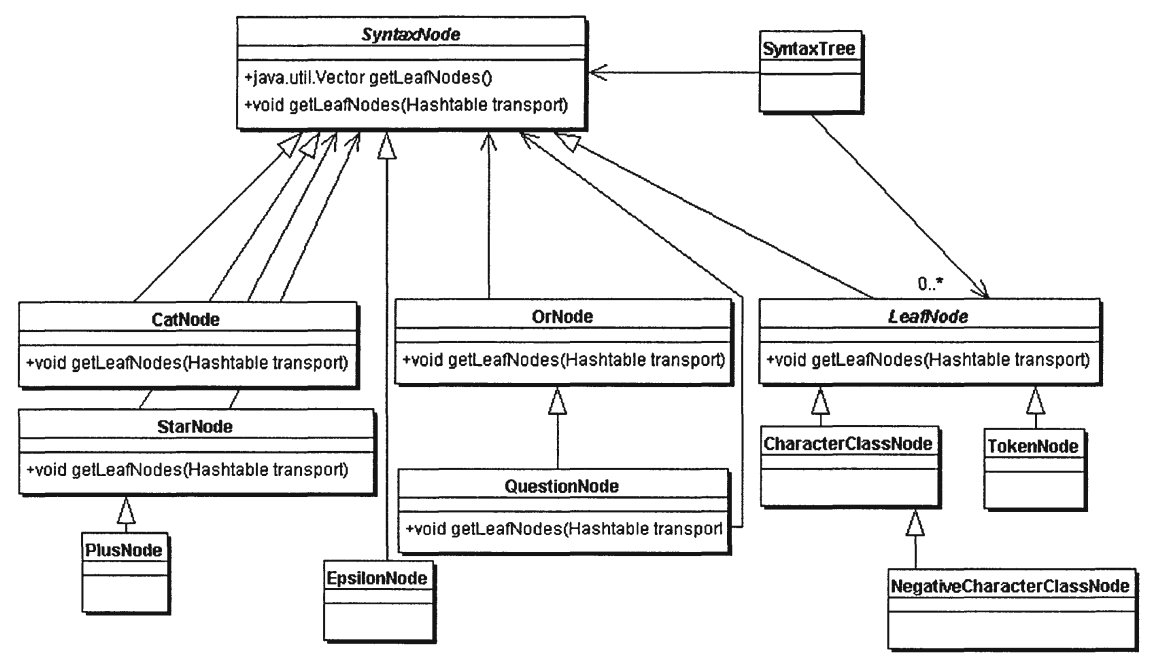

Figure 9. AWK part of ORO after the extension

\subsection{General remarks and evaluation of criteria}

Several other case studies have been processed, but not all of them can be presented here in detail. It has been observed, that the method works best with tree like structures, that do not have any cycles.

Now the criteria from section 1 are revisited and it is checked if and how the method satisfies them.

- Scalability: The method scales well. It can be used with small and large systems. Using the AP concepts in the new approach the developer can focus on a local part of the system. Having to maintain a global view would be too complex.

- Universality: As claimed in the beginning, the method itself can deal with every kind of object-oriented source code, but the current implementation can only process Java code. This has been achieved by modifications of the AP model and the way that new functionality is described in a metaprogram apart the existing code and new source code is generated then. An implementation for $\mathrm{C}++$ would additionally require support for templates. But this can be achieved by figuring out in a precalculating step which types are used while creating instances of the templates. 
- Degree of abstraction: Extensions can be specified on a more abstract level by leaving out implementation details like navigation and container-classes. So the method fulfill this criterion since no detailed white-box knowledge is necessary during specification and execution.

- Expressiveness: Compared to the expressiveness of standard procedures and considering the amount of written lines of code, the new approach generally is better. This results from the generated source code pieces for navigation and method bodies, enabled by specifying the involved classes with a navigation directive.

- Source code quality: If the source code is expandable and readable before an application of the new method, it remains in that state, since all the method does is generating additional methods and method bodies. Afterwards the code can be processed with every other method or tool.

\section{Summary and future work}

The goal of this work was developing a method that is suitable for performing a special kind of extensions to object-oriented systems during forward- and re-engineering. The extensions are characterized by affecting the interface of an existing subsystem and several other classes being part of it. Some of the classes only have to perform method calls, while other classes implement the functionality.

Performing these extensions with state of the art methods is very hard and error-prone, since detailed white-box knowledge must be maintained during the process.

Therefore the existing AP approach has been made usable in evolutionary software development, by modifying and extending it, in order to specify the extensions apart from the existing code on a more abstract level. So detailed white-box knowledge is not necessary any more during specification.

Currently several ideas exist how the approach can be further evolved. One point concerns the so called "robustness versus structural changes", which means that AP propagation patterns might be reused, if the structure of a system changes within several restrictions. To have this property in the new approach, it must be possible to undo the results of an application of the method, which is currently not possible.

Another nice feature to have would be a graphical mechanism for specifying navigation directives, to give the user a direct impression which classes he selects. This would make the specification of propagation patterns much easier. 
One concern which should be also taken care of in future are possible side effects of the method. Since it only generates additional code, nothing is going to be deleted, but since the user gains full control over class attributes, it cannot be ensured that existing methods will behave like they did before the method has been applied.

\section{References}

[1] Ole Agesen. Constraint-based type inference and parametric polymorphism. In First International Static Analysis Symposium. Springer Verlag, 1994.

[2] Kent Beck. Extreme Programming Explained: Embrace Change. Addison Wesley, 1999.

[3] Krzysztof Czarnecki and Ulrich W. Eisenecker. Generative Programming. Addison-Wesley, 2000.

[4] Martin Fowler. Refactoring: Improving the Design of Existing Code. Addison Wesley, 1999.

[5] E. Gamma, R. Helm, R. Johnson, and J. Vlissides. Design Patterns: Elements of Reusable Object-Oriented Software. Addison Wesley, 1994.

[6] W. Hursch. Should superclasses be abstract, 1994.

[7] Palo Alto Research Center Incorporated. AspectJ - aspect-oriented programming (AOP) for Java. http://aspectj.org/, 2002.

[8] Gregor Kiczales, John Lamping, Anurag Menhdhekar, Chris Maeda, Cristina Lopes, Jean-Marc Loingtier, and John Irwin. Aspect-oriented programming. In Mehmet Akşit and Satoshi Matsuoka, editors, ECOOP '97 - Object-Oriented Programming 11th European Conference, Jyväskylä, Finland, volume 1241, pages 220-242. Springer-Verlag, New York, NY, 1997.

[9] Karl Lieberherr. Demeter Forschungsgruppe. http://www.ccs.neu.edu/research/demeter/.

[10] Karl Lieberherr. Adaptive Object-Oriented Software: The Demeter Method. PWS Publishing Company, 1995.

[11] Karl Lieberherr and John Sung. TraversalJ. http://www.ccs.neu.edu/home/lieber/com1205/w02/project/ com1205Project_w02.doc.

[12] William F. Opdyke. Refactoring Object-Oriented Frameworks. PhD thesis, University of Illinois at Urbana-Champaign, 1992.

[13] Doug Orleans and Karl Lieberherr. DJ: Dynamic adaptive programming in Java. http://www.ccs.neu.edu/research/demeter/biblio/DJ-reflection.html, 2001.

[14] Jens Palsberg and Michael Schwartzbach. Object-oriented type inference. Technical report, DAIMI PB-345, Computer Science Department, Aarhus University, Mrz 1991.

[15] Ian Sommerville. Software Engineering. Addison Wesley, 1996.

[16] Michael Werner. Facilitating Schema Evolution with Automatic Program Transformations. PhD thesis, Northeastern University, 1999. 
[17] Norman Wilde and Ross Huit. Maintenance support for object-oriented programs. In IEEE Transactions on software engineering, volume 18, December 1992. 